\title{
Effect of CALR and JAK2 mutations on the clinical and hematological phenotypes of the disease in patients with myelofibrosis - long-term experience from a single center
}

\author{
M. PALOVA*, T. SZOTKOWSKI, A. HLUSI, K. INDRAK, J. NAVRATILOVA, M. DIVOKA, T. PAPAJIK \\ Department of Hemato-Oncology, Faculty of Medicine and Dentistry, Palacky University Olomouc and University Hospital Olomouc \\ ${ }^{*}$ Correspondence: miroslava.palova@fnol.cz
}

Received April 26, 2017/ Accepted June 28, 2017

\begin{abstract}
Primary myelofibrosis (PMF) is a chronic clonal myeloid disorder. Together with essential thrombocythemia (ET) and polycythemia vera (PV), it belongs to a group of Philadelphia chromosome-negative myeloproliferative neoplasms. An integral part of laboratory tests carried out in this disease group is detecting the presence of mutations in the Janus kinase 2 gene at position 617 (JAK2 V617F) and in the gene encoding for the receptor for thrombopoietin (myeloproliferative leukemia virus oncogene, $M P L$ ) found in approximately $60 \%$ of PMF patients. The discovery of mutations affecting exon 9 of the calreticulin (CALR) gene was of great benefit to the diagnosis of the diseases in JAK2 V617F and MPL unmutated cases. This is a study of the effect of a mutation in the CALR gene on the clinical course in patients with primary, post-ET and post-PV myelofibrosis. Analysis of 66 patients (54.5\% JAK2 V617F; 34.8\% CALR; 6.1\% MPL; 3.0\% triple negative; $1.5 \%$ coincidence of $C A L R$ and $J A K 2 \mathrm{~V} 617 \mathrm{~F}$ ) confirmed a different phenotype of the disease in CALR-mutated patients as compared with $C A L R$-unmutated individuals. Those with $C A L R$ mutation were significantly younger and had borderline higher platelet counts, less pronounced splenomegaly and less frequent B symptoms at diagnosis. The study suggests that the driver mutation types define variations in the biological basis, clinical manifestations and course of the disease. The presence of CALR mutation has been shown to be an independent prognostic favorable factor. Careful risk stratification of these patients is of great importance to adequate therapeutic decision-making and aids in selecting high-risk patients eligible for allogeneic hematopoietic stem cell transplantation which continues to be the only treatment modality for myelofibrosis having curative potential.
\end{abstract}

Key words: myeloproliferative disease, myelofibrosis, calreticulin (CALR), JAK2 V617F

Primary myelofibrosis (PMF) is classified among chronic Philadelphia chromosome-negative myeloproliferative neoplasms (Ph-MPN), together with essential thrombocythemia (ET) and polycythemia vera (PV); these may progress to myelofibrosis (MF) [1]. The incidence of PMF is 1.5 cases per 100,000 population per year; the median age at diagnosis is 67 years. Approximately $17 \%$ of patients are diagnosed before the age of $50[2,3]$. The incidence of PV is higher in males than in females ( 2.8 vs. 1.3 cases per 100,000 population per year). The cumulative risk of progression to post-PV MF is $6 \%$ after 10 years and $26 \%$ after 20 years of disease duration $[4,5]$. The incidence of ET is 2.5 cases per 100,000 population per year; the cumulative risk of transformation to post-ET MF is $9 \%$ after 15 years of disease duration $[6,7]$.

Of key importance in the pathogenesis of PMF is dysregulation or constitutional activation of the Janus kinase-signal transducer and activator of transcription (JAK/STAT) signaling pathway, leading to STAT protein autophosphorylation. The phosphorylated forms of STAT proteins translocate into the cell nucleus and participate in activation of transcription of genes regulating cell proliferation, differentiation and apoptosis [8]. This results in cytokine-independent proliferation of hematopoietic stem cells and activation of other signaling pathways involving STAT3, STAT5, MAPK, ERK and PI3K-AKT $[8,9]$. The constitutional activation of the JAK/STAT pathway may be caused either directly by somatic mutation of the JAK2 gene at position V617F or indirectly by mutations in the gene with a regulatory effect on this signaling pathway (MPL). Mutation in the calreticulin $(C A L R)$ gene is also associated with STAT protein activation, but the exact mechanism remains unknown. The signaling pathway activation results in clonal proliferation of atypical 
megakaryocytes with more intense production of cytokines and growth factors, leading to enhanced proliferation of non-clonal fibroblasts with subsequent MF. This results in the development of cytopenia, extramedullary hematopoiesis, hepatosplenomegaly and constitutional symptoms such as elevated body temperature, night sweats or weight loss [10-13].

The JAK2 V617F somatic mutation, discovered in 2005, led to a breakthrough in the classification, diagnosis and understanding of Ph-MPN [14]. However, in only $50-60 \%$ of PMF cases the JAK2 V617F mutation is detected; in another $5-8 \%$ of cases, mutation in codons 515 or 505 of the gene encoding for the receptor for thrombopoietin $(M P L)$ is detected $[12,13]$.

After 2003, when Klampfl et al. and Nangalia et al. described recurrent somatic mutations affecting exon 9 of the CALR gene occurring in most JAK2 and MPL unmutated patients with PMF, the proportion of patients with a known molecular marker of clonality increased to $80-85 \%[10,13]$. The CALR gene encodes for calreticulin, an endoplasmic reticulum chaperone capable of binding $\mathrm{Ca}^{2+}$. Mutations in the CALR gene are a rather heterogeneous group comprising at least 50 different mutations such as short deletions, insertions or substitutions located in exon 9 of the CALR gene on chromosome 19. The two most common (in $80 \%$ of cases) mutations are 52-bp deletion (CALR del52/type1) and 5-bp insertion (CALR ins5/type2). Most of them cause a shift in the reading frame, with an alternative reading frame leading to the production of mutant proteins. It is assumed that mutations influence the subcellular localization, stability or function of calreticulin $[15,16]$. The present study aimed to determine the association between the mutational status and clinical or laboratory phenotype of the disease in patients with histologically confirmed MF.

\section{Patients and methods}

Patients. Included in the study were all 66 patients diagnosed in our center between 1998 and 2016. There were 45 patients diagnosed with PMF (of whom 13 were in the prefibrotic phase of PMF at the time of diagnosis), 10 individuals with post-PV MF and 11 patients with post-ET MF. At the time of diagnosis, all participants met the 2008 WHO classification criteria. The sample comprised 30 males and 36 females with a median age of 55.5 years (range 18-77 years). Written informed consent was obtained from all participants before their genetic material (DNA) was processed and analyzed. Laboratory parameters and clinical data from the time of diagnosis were collected retrospectively. Objectively confirmed thrombotic and hemorrhagic complications were recorded if these occurred at the time of diagnosis or during the follow-up. Thrombotic complications included venous thrombosis (deep venous thrombosis, pulmonary embolism, splanchnic venous thrombosis or cerebral venous sinus thrombosis) and arterial thrombosis (transient ischemic attack, ischemic stroke, acute myocardial infarction and peripheral arterial vascular complications); hemorrhagic complications were gastrointestinal bleeding, hematuria, hemorrhagic stroke and significant bleeding during medical or dental surgery. Transformation to acute leukemia (AL) was diagnosed in accordance with the WHO criteria. Constitutional symptoms included elevated body temperature, weight loss and night sweats. Clinical and laboratory parameters were systematically compared with the patients' mutational status.

Detection of somatic mutations. All patients were tested for the presence of JAK2 V617F, MPL and CALR gene mutations. All analyses were performed using DNA isolated from peripheral white blood cells (WBCs). In all patients, a mutation in codon 617 of the JAK2 gene was studied by allele-specific polymerase chain reaction (AS-PCR). In case of positive findings, real-time PCR was used for quantification and subsequent calculation of percentages of the mutated allele [17]. In all participants, screening of exon 9 of the CALR gene was performed using fragment analysis. In positive cases, the exact mutation type was identified by Sanger sequencing $[10,18]$. Mutations of the MPL gene (S505N and W515) were studied by AS-PCR and fragment analysis; the detected mutations were confirmed by Sanger sequencing [12].

Statistical analysis. Relationships between categorical variables were assessed by means of independence tests in contingency tables. For large enough patient counts, chi-square asymptotic test of independence was used, otherwise Fisher's exact factorial test was used. Differences in continuous variables among patient groups were visualized by means of standard box plots and tested by the KruskalWallis test. The test compares the means of the samples in the respective groups, and returns the p-value for the null hypothesis that all samples are drawn from the same population. The Kruskal-Wallis test is a non-parametric vision of the classical one-way ANOVA, and an extension of the Wilcoxon rank sum test to more than two groups. For survival analysis, the non-parametric Kaplan-Meier estimator was used to visualize the survival curves. Difference in survival among groups was tested by means of the log-rank test. All tests were performed at the 0.05 level of significance. The statistical analyses were performed in MATLAB and Statistics Toolbox Release 2013b (MathWorks), and STATISTICA 12 (StatSoft).

\section{Results}

At the time of analysis, the median follow-up of patients was 46 months (range 2-221 months); thirteen (20.3\%) patients died after a median of 42 months (range 13-173 months) from diagnosis. Three patients progressed to $\mathrm{AL}$ after a median of 24 months from diagnosis of high risk PMF or from transformation to post-PV MF. Two patients with AL died after 4-6 months from progression to AL due to infectious complications. 


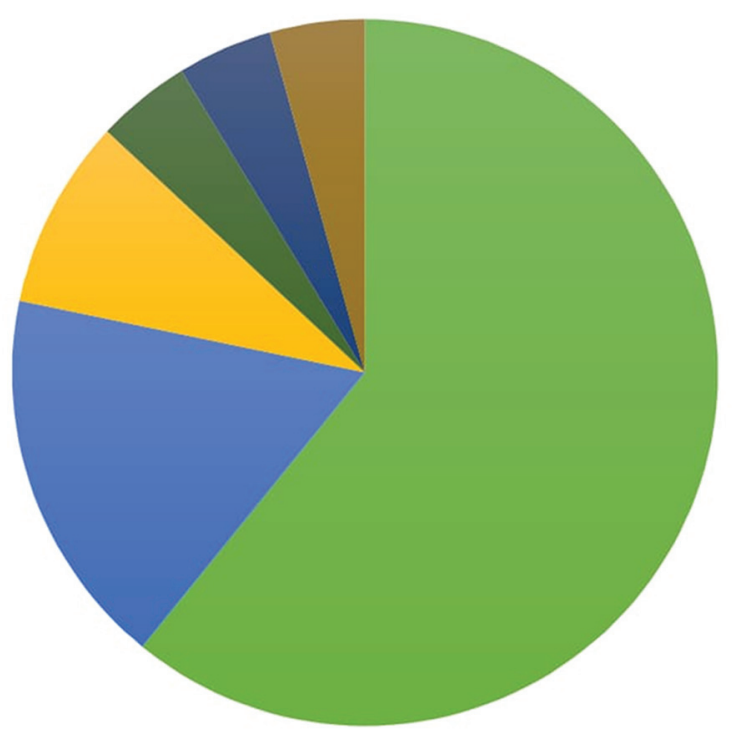

del52 $\square$ ins5 $\square$ ins $\square \operatorname{del} 3 \square \operatorname{del} 46 \square \operatorname{del} 19$

Figure 1. Distribution of types of mutation in the CALR gene. In the sample of 66 patients with PMF, post-ET myelofibrosis and post-PV myelofibrosis, mutation in the CALR gene were found in $34.8 \%$. The distribution of CALR mutations types: type 1 mutation $60.8 \%$, type 2 mutation $17.4 \%$ and other types $21.8 \%$ (see the graph). PMF - primary myelofibrosis; ET - essential thrombocythemia; $\mathrm{PV}$ - polycythemia vera; CALR - calreticulin.

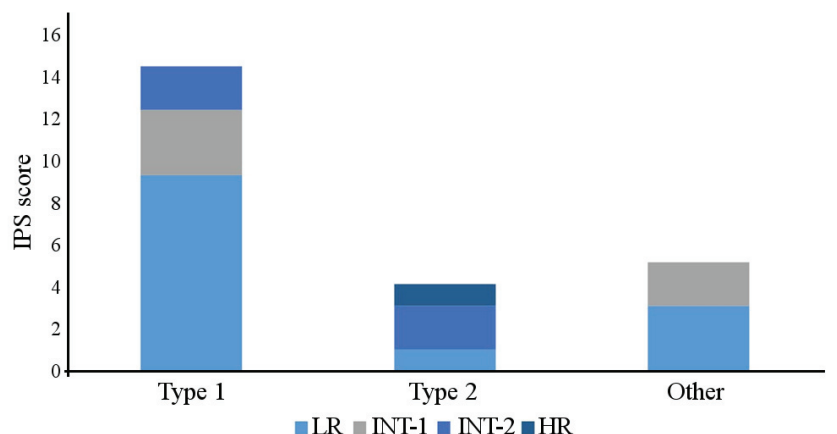

Figure 2. Distribution of IPSS scores at diagnosis depending on the CALR mutation types. The distribution of IPSS scores at diagnosis in the sample of 66 patients with PMF, post-ET myelofibrosis and post-PV myelofibrosis depending on types of mutation in the CALR gene. PMF - primary myelofibrosis; ET - essential thrombocythemia; PV - polycythemia vera; IPSS - International Prognostic Scoring System; CALR - calreticulin.

The driver mutations were distributed as follows: JAK2 V617F 36 cases (54.5\%), CALR (exon 9) 23 cases (34.8\%) and MPL W515 4 cases (6.1\%). In the PMF subgroup, the distribution of driver mutations did not differ from the whole group. In one patient, both JAK2 V617F and CALR mutations were detected; by contrast, two patients were triple-negative. Among the CALR-mutated cases, $60.8 \%$ were of type 1 (14/23), 17.4\% were of type 2 (4/23) and $21.8 \%$ (5/23) were of less frequent types (Figure 1). The clinical phenotype at the

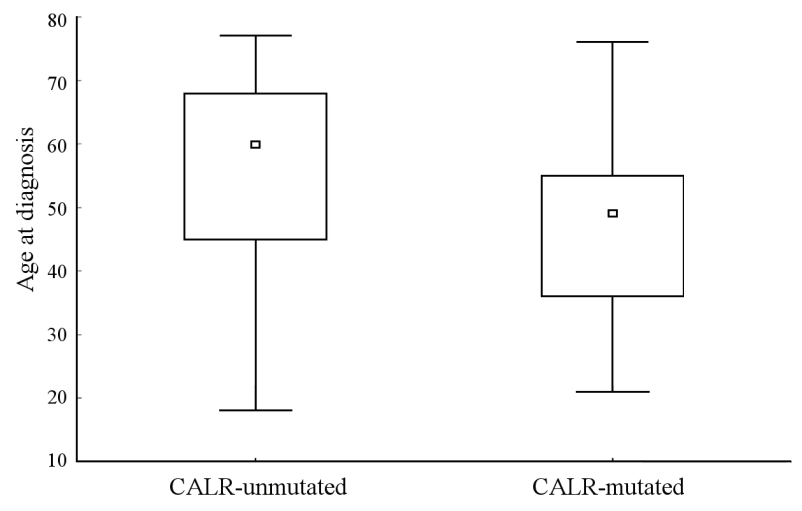

Figure 3. Age at diagnosis depending on the mutational status. In the sample of 66 patients with PMF, post-ET myelofibrosis and post-PV myelofibrosis (24 CALR-mutated, 42 CALR-unmutated), CALR-mutated patients were significantly younger at diagnosis as compared to CALRunmutated individuals $(\mathrm{p}=0.009)$. PMF - primary myelofibrosis; $\mathrm{ET}$ - essential thrombocythemia; PV - polycythemia vera; CALR - calreticulin.

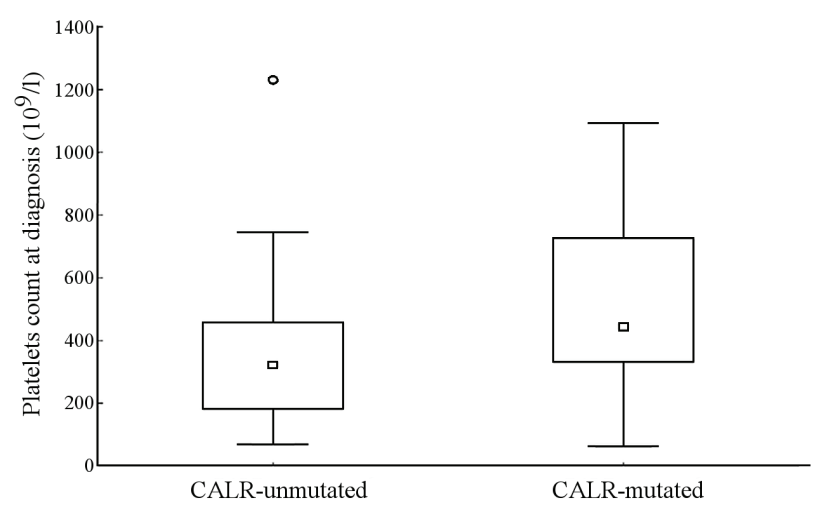

Figure 4. Platelet count at diagnosis depending on the mutational status. In the sample of 66 patients with PMF, post-ET myelofibrosis and postPV myelofibrosis (24 CALR-mutated, 42 CALR-unmutated), CALRmutated patients had significantly higher platelet counts $\left(\times 10^{9} / \mathrm{L}\right)$ at diagnosis as compared to CALR-unmutated individuals $(\mathrm{p}=\mathbf{0 . 0 5 1})$. PMF - primary myelofibrosis; ET - essential thrombocythemia; PV - polycythemia vera; CALR - calreticulin.

time of diagnosis is summarized in Table 1. The distribution of International Prognostic Scoring System (IPSS) scores at diagnosis depending on types of mutation in the calreticulin (CALR) gene is summarized in Figure 2.

CALR-mutated patients were found to be significantly younger at diagnosis (49 vs 59 years, $\mathrm{p}=0.009$, Figure 3) and had borderline higher platelet counts $\left(445 \times 10^{9} / \mathrm{L}\right.$ vs $312 \times 10^{9} / \mathrm{L}, \mathrm{p}=0.051$, Figure 4$)$, much smaller spleen size $(2 \mathrm{~cm}$ vs $10 \mathrm{~cm}$ below the left costal margin, $\mathrm{p}=0.005$, Figure 5) and less frequent B symptoms (4.5\% vs $56.5 \%$, $\mathrm{p}=0.02)$ at diagnosis as compared with CALR-unmutated patients. CALR-mutated patients also had lower WBC counts at diagnosis but the difference was not statistically significant. Similarly, there were no statistical differences in the levels of hemoglobin ( $\mathrm{Hb})$, hematocrit (Hct), blasts in the 
Table 1. Clinical and laboratory phenotypes of patients with myelofibrosis at diagnosis depending on the mutational status (JAK2 V617F, CALR, MPL).

\begin{tabular}{|c|c|c|c|}
\hline & $J A K 2 \mathrm{~V} 617 \mathrm{~F}$ & CALR & MPL \\
\hline Presence of mutation (\%) & 54.5 & 34.8 & 6.1 \\
\hline Males/ females & $13 / 23$ & $12 / 11$ & $3 / 1$ \\
\hline Median age at diagnosis (years) & $59(18-77)$ & $49(23-76)$ & $62.5(44-68)$ \\
\hline Median $\mathrm{Hb}$ level at diagnosis $(\mathrm{g} / \mathrm{L})$ & $127(78 / 155)$ & $120(86-148)$ & $99.5(90-110)$ \\
\hline Median WBC count at diagnosis $\left(\times 10^{9} / \mathrm{L}\right)$ & $11.8(4.5-37.7)$ & $8.3(1.5-26.9)$ & $5.9(1.8-19.2)$ \\
\hline Median platelet count at diagnosis $\left(\times 10^{9} / \mathrm{L}\right)$ & $312(74-1233)$ & $445(62-1093)$ & $179(68-342)$ \\
\hline Median circulating blasts (\%) & $0(0-4)$ & $0(0-4)$ & $1(0-2)$ \\
\hline Median LDH level at diagnosis $(\mu \mathrm{kat} / \mathrm{L})$ & $7(3-30)$ & $5.78(2.9-19.7)$ & $11.2(7.9-20.1)$ \\
\hline Splenomegaly at diagnosis (median $\mathrm{cm}$ below the left costal margin) & $10(0-30)$ & $2(0-10)$ & $6.5(3-10)$ \\
\hline Presence of B symptoms at diagnosis (\%) & 56.5 & 4.5 & 0 \\
\hline
\end{tabular}

\section{IPSS at diagnosis}

Low risk

Intermediate-1 risk

Intermediate-2 risk

High risk

Total number of patients no. of patients

11

17

8

0

36 no. of patients

12

7

4

0

23 no. of patients

0

4

0

0

4

Comparison of the clinical and laboratory findings and International Prognostic Scoring System (IPSS) scores at diagnosis depending on the mutational status (JAK2 V617/ CALR/ MPL mutated patients). The total number of patients was 66: 36 JAK2V617F positive, 23 CALR positive, 4 MPL positive, 2 triple negative. In one case both JAK2V617F and CALR mutations were detected; 1B symptoms: elevated body temperature, weight loss and night sweats; WBC - white blood cells

Table 2. Distribution of driver mutations in patients with thrombotic complications.

\begin{tabular}{lcccc}
\hline & CALR & Triple negative & JAK2 V617F & MPL \\
Venous thrombosis (no. of patients) & 3 & 1 & 8 \\
Arterial thrombosis (no. of patients) & 3 & 0 & 2 \\
\hline
\end{tabular}

peripheral blood and lactate dehydrogenase (LDH) between the two subgroups.

Thrombotic complications developed in $27 \%$ of patients; $72 \%$ of those cases had venous thrombotic complications and the others had arterial complications. Hemorrhagic complications were documented in $1.5 \%$ of patients with concomitant moderate thrombocytopenia. In $46 \%$ of patients with venous thrombosis and $60 \%$ of those with arterial thrombosis, additional risk factors were identified, most frequently acquired protein $\mathrm{S}$ deficiency, protein $\mathrm{C}$ deficiency and the presence of lupus anticoagulant. The distribution of driver mutations in patients with thrombotic complications is shown in Table 2. Twenty-three percent of patients had a recurrent thrombosis. In all cases, the recurrence occurred in the venous district. This recurrent thrombosis was documented in patients who discontinued oral anticoagulation therapy with vitamin K antagonists (VKA) or in patients treated with VKA, but with non-therapeutic INR (the International normalized ratio). All of these patients had one of the above mentioned additional risk factor.

In JAK2 V617F-positive patients, the effects of the mutated allele burden on the clinical and laboratory phenotypes of the disease were studied. The median allele burden was 53\% (range, 7.7-99\%). Patients with a JAK2 V617F allele burden of more than $50 \%$ were found to have significantly more

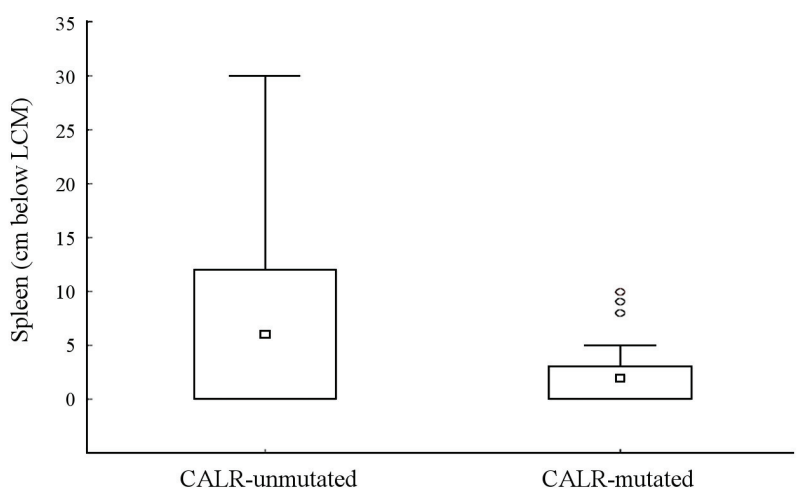

Figure 5. Presence of splenomegaly at diagnosis depending on the mutational status. In the sample of 66 patients with PMF, post-ET myelofibrosis and post-PV myelofibrosis (24 CALR-mutated, 42 CALR-unmutated), CALR-mutated patients had significantly less pronounced splenomegaly (cm below LCM) at diagnosis as compared to CALR-unmutated individuals $(p=0.005)$. PMF - primary myelofibrosis; ET - essential thrombocythemia; PV - polycythemia vera; CALR - calreticulin, LCM - left costal margin.

pronounced splenomegaly $(12 \mathrm{~cm}$ vs $0 \mathrm{~cm}$ below the left costal margin, $\mathrm{p}=0.0001$, Figure 6 ) and higher WBC counts $\left(16.7 \times 10^{9} / \mathrm{L}\right.$ vs $8.8 \times 10^{9} / \mathrm{L}, \mathrm{p}=0.003$, Figure 7$)$ at diagnosis. Differences in the other parameters ( $\mathrm{Hb}$, Hct, blasts in the 


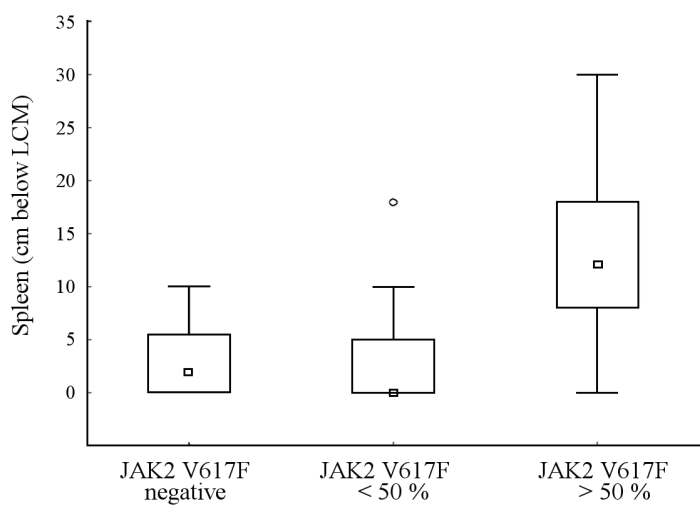

Figure 6. Presence of splenomegaly at diagnosis depending on the JAK2 V617F allele burden. In the sample of 66 patients with MF, 29 individuals were JAK2 V617F negative, 17 patients with a JAK2 V617F allele burden $>50 \%$, 17 patients with a JAK2 V617F allele burden $<50 \%$, in three patients the quantification was not performed. Significantly more pronounced splenomegaly (cm below LCM) at diagnosis was found in patients with a percentage of the JAK2 V617F mutated allele of more than $50 \%(p=0.0001)$. MF - myelofibrosis; JAK2 V617F - mutation in the Janus kinase 2 gene at position 617; LCM - left costal margin.

Table 3. International Prognostic Scoring System (IPSS)/Dynamic International Prognostic Scoring System (DIPSS) Plus prognostic models.

\begin{tabular}{lcc}
\hline Risk factors & IPSS & DIPSS Plus \\
\hline Age $>65$ years & 1 & 1 \\
B symptoms $^{1}$ & 1 & 1 \\
Hemoglobin $<100 \mathrm{~g} / \mathrm{L}$ & 1 & 1 \\
White blood cells $>25 \times 10^{9} / \mathrm{L}$ & 1 & 1 \\
Circulating blasts $\geq 1 \%$ & 1 & 1 \\
Platelets $<100 \times 10^{9} / \mathrm{L}$ & & 1 \\
Transfusion dependency & & 1 \\
Unfavorable karyotype ${ }^{2}$ & & 1 \\
& & \\
Risk group & OS (months) & OS (months) \\
Low risk & 135 & 185 \\
Intermediate-1 risk & 95 & 78 \\
Intermediate-2 risk & 48 & 35 \\
High risk & 27 & 16 \\
\hline
\end{tabular}

OS - overall survival; ${ }^{1} \mathrm{~B}$ symptoms: elevated body temperature, weight loss and night sweats; ${ }^{2}$ Unfavorable karyotype: complex karyotype, $+8,-7 / 7 \mathrm{q}$-, $\mathrm{i}(17 \mathrm{q})$, inv(3), -5/5q-, 12p-, 11q23 rearrangement; ${ }^{3}$ IPSS: low risk (0 points), intermediate- 1 risk ( 1 point), intermediate- 2 risk ( 2 points), high risk (3-5 points). DIPSS Plus: low risk (0 points), intermediate- 1 risk (1 point), intermediate- 2 risk (2-3 points), high risk ( $\geq 4$ points)

Table 4. Risk stratification in MF (Tefferi et al., 2014).

\begin{tabular}{lc}
\hline Mutational status & Overall survival (years) \\
\hline CALR+/ASXL1- & 10.4 \\
CALR+/ASXL1+; CALR-/ASXL1- & 5.8 \\
CALR-/ASXL1+ & 2.3 \\
\hline
\end{tabular}

Risk stratification of patients with MF based on the presence of mutations in the calreticulin $(C A L R)$ or ASXL1 gene.

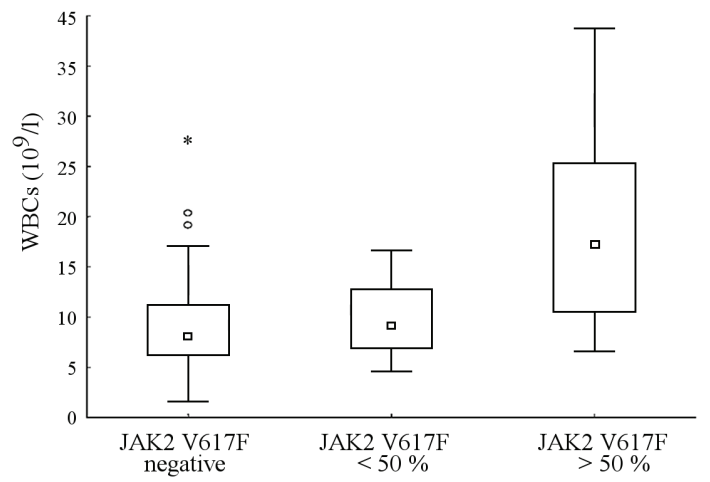

Figure 7. White blood cell count at diagnosis depending on the JAK2 V617F allele burden. In the sample of 66 patients with MF, 29 individuals were JAK2 V617F negative, 17 patients with a JAK2 V617F allele burden $>50 \%, 17$ patients with a JAK2 V617F allele burden $<50 \%$, in three patients the quantification was not performed. Significantly higher white blood cell counts (WBCs $\left.\times 10^{9} / \mathrm{L}\right)$ at diagnosis were found in patients with a percentage of the JAK2 V617F mutated allele of more than $50 \%$ $(\mathrm{p}=0.003)$. MF - myelofibrosis; JAK2 V617F - mutation in the Janus kinase 2 gene at position 617; WBCs - white blood cells.

peripheral blood, platelets and $\mathrm{LDH}$ ) were not statistically significant.

The median OS rates were 47 months and 45 months in $C A L R$-mutated and CALR-unmutated patients, respectively.

\section{Discussion}

The percentage of driver mutations in the present study is consistent with those reported by other authors $[10,13$, 19]. This cohort of CALR-mutated MF patients is characterized by a significantly younger age, less pronounced splenomegaly, less frequent $\mathrm{B}$ symptoms and borderline higher platelet count at the time of diagnosis. WBC counts were also lower in CALR-mutated cases (statistical significance not achieved). The findings are consistent with previous analyses showing significantly positive effects of the presence of the CALR mutation [20-23]. There were no significant differences in the other tested parameters. In addition, some authors (e.g. Rumi et al.) identified lower cumulative incidence rates of anemia in CALR-mutated individuals [20]. Consistently with other reports, the present study confirmed comparable distribution of CALR mutation variants in $\mathrm{MF}$, with type 1 (CALR del52) being the most common mutation. Given the generally relatively small number of $C A L R$ mutated patients, relevant statistical assessment of the impact of CALR mutation types on disease phenotypes could not be performed. However, multicenter studies suggest that a more favorable phenotype and prognosis is associated with only type $1 C A L R$ mutations $[21,24,25]$. A recent study by Tefferi et al. showed different laboratory and clinical findings in MF with type 2 CALR mutation (higher WBC count, higher percentage of circulating blasts, more pronounced splenomegaly and OS comparable with JAK2 V617F-mutated cases). 
The differences are likely to be due to different pathogenetic mechanisms of individual CALR mutations [24]. Recently, investigators also reported relevant structural and functional differences between proteins encoded by various $C A L R$ gene variations [26]. However, relatively low numbers of MF cases with type 2 CALR mutations in studies published so far prevent deeper understanding of how the CALR mutation type affects the OS. A Mayo Clinic study showed the poorest prognosis with a median OS of 2.5 years in triple-negative patients [27]. The rare concomitant presence of mutations in the JAK2 and CALR gene may reflect the presence of two different clones in a patient [27-29].

Consistently with data published by other authors, subanalysis of JAK2 V617F mutated patients confirmed the association of a mutated allele burden of more than 50\% with clinical and laboratory manifestations of the disease (significantly more pronounced splenomegaly and higher WBC count at diagnosis) $[30,31]$. The median percentage of mutated alleles was $53 \%$. It must be noted that the two studies showed correlations between shorter OS in PMF and a lower JAK2 V617F allele burden. The exact biological cause of this remains unclear, but it is assumed that another V617F-negative clone is present that is responsible for the more aggressive disease course [30,32].

In the present study, thrombotic complications were diagnosed in $27 \%$ of patients, with venous thrombosis being more common. In $46 \%$ of patients with venous thrombosis and $60 \%$ of those with arterial thrombosis, additional risk factors were documented. Twenty-three percent of patients had a recurrent thrombosis after stopping VKA or in case of non-therapeutic INR. Due to the relatively low number of events recorded, a relevant statistical analysis of the effect of the mutational status on the risk of thrombosis could not be performed. However, Rumi et al. claimed that although CALR mutation is associated with higher platelet counts in MF, the risk of thrombosis is lower as compared with JAK2 V617Fmutated individuals. The risk of thromboembolic complications remains lower in patients carrying both type 1 and type 2 mutations of the CALR gene [20]. Due to the small number of recorded events, a relevant statistical analysis of difference in OS between the subgroups of CALR-mutated patients and their unmutated counterparts could not be performed. However, other multicenter studies showed that CALR mutation is associated with better OS of patients, independent of their International Prognostic Scoring System/ Dynamic International Prognostic Scoring System scores (Table 3) [20, 27]. Recent studies identified other subclonal mutations in the ASXL1, SRSF2, EZH2 and IDH1/2 genes, associated with poor prognosis and a higher risk of leukemic transformation [22, 33, 34]. At the time of performing this study, assessment of these mutations was not available for routine clinical practice. Tefferi et al. published risk stratification of patients based on CALR/ASXL1 mutational status, with prolonged OS in $C A L R+/ A S X L 1-$ patients as compared with CALR-/ASXL1+ patients (Table 4) [35].
Conclusion. Despite its limitations, the present retrospective analysis showed differences in the clinical course of the disease, suggesting differences in its biological basis depending on the type of mutation. All driver mutations lead to persistent activation of the JAK/STAT signaling pathway, but are likely to differ by their specific effect on other metabolic processes [15]. The present analysis confirmed the observation that the presence of CALR mutation significantly influences the clinical phenotype and course of the disease. Analyzing CALR mutation in routine clinical practice is important for more accurate assessment of an individual risk of the disease and determination of its clonality. The current risk stratification in MF is based on demographic, clinical and hematological parameters [36-38]. Our experiences confirm the previously reported data on the role of driver mutations determining various clinical manifestations and course of MPN. Given the low incidence of the disease, more valid data may only be provided by multicenter collaboration and assembling a large enough cohort of patients. The aim is to develop a prognostic model that takes into account the molecular profile and predicts the treatment response. The presence of CALR mutation has a clearly positive effect on the prognosis of MF patients and should be taken into consideration not only in risk stratification of patients but also in clinical studies testing the effectiveness of novel treatment modalities [20, 23]. Last but not least, it is also necessary to elucidate the exact mechanism of CALR mutation in the pathogenesis of MF and thus identify a new potential therapeutic goal.

Acknowledgements: The study was supported by the Palacký University grant project IGA_LF_2017_007. Special thanks to Tomáš Fürst for performing the statistical analysis.

\section{References}

[1] TEFFERI A. Primary myelofibrosis: 2017 update on diagnosis, risk-stratification, and management. Am J Hematol 2016; 91: 1262-1271. doi: 10.1002/ajh.24592

[2] MESA RA, SILVERSTEIN MN, JACOBSEN SJ, WOLLAN PC, TEFFERI A. Population-based incidence and survival figures in essential thrombocythemia and agnogenic myeloid metaplasia: an Olmsted County Study, 1976-1995. Am J Hematol 1999; 61: 10-15.

[3] VISANI G, FINELLI C, CASTELLI U, PETTI MC, RICCI $P$ et al. Myelofibrosis with myeloid metaplasia: clinical and haematological parameters predicting survival in a series of 133 patients. Br J Haematol 1990; 75: 4-9.

[4] ANÍA BJ, SUMAN VJ, SOBELL JL, CODD MB, SILVERSTEIN MN et al. Trends in the incidence of polycythemia vera among Olmsted County, Minnesota residents, 19351989. Am J Hematol 1994; 47: 89-93.

[5] BONICELLI G, ABDULKARIM K, MOUNIER M, JOHANSSON P, ROSSI C et al. Leucocytosis and thrombosis at diagnosis are associated with poor survival in polycythaemia vera: a population-based study of 327 patients. Br J Haematol 2013; 160: 251-254. doi: 10.1111/bjh.12117 
[6] MOULARD O, MEHTA J, FRYZEK J, OLIVARES R, IQBAL $\mathrm{U}$ et al. Epidemiology of myelofibrosis, essential thrombocythemia, and polycythemia vera in the European Union. Eur J Haematol 2014; 92: 289-297. doi: 10.1111/ejh.12256

[7] BARBUI T, THIELE J, PASSAMONTI F, RUMI E, BOVERI E et al. Survival and disease progression in essential thrombocythemia are significantly influenced by accurate morphologic diagnosis: an international study. J Clin Oncol 2011; 29: 3179-3184. doi: 10.1200/JCO.2010.34.5298

[8] REILLY JT, MCMULLIN MF, BEER PA, BUTT N, CONEALLY E et al. Guideline for the diagnosis and management of myelofibrosis. Br J Haematol 2012; 158: 453-471. doi: 10.1111/j.1365-2141.2012.09179.x

[9] GUGLIELMELLI P, BAROSI G, RAMBALDI A, MARCHIOLI R, MASCIULLI A et al. Safety and efficacy of everolimus, a mTOR inhibitor, as single agent in a phase $1 / 2$ study in patients with myelofibrosis. Blood 2011; 118: 2069-2076. doi: 10.1182/blood-2011-01-330563

[10] KLAMPFL T, GISSLINGER H, HARUTYUNYAN AS, NIVARTHI H, RUMI E et al. Somatic mutations of calreticulin in myeloproliferative neoplasms. N Engl J Med 2013; 369: 2379-2390. doi: 10.1056/NEJMoa1311347

[11] TEFFERI A. Pathogenesis of myelofibrosis with myeloid metaplasia. J Clin Oncol 2005; 23: 8520-8530. doi: 10.1200/ JCO.2004.00.9316

[12] PIKMAN Y, LEE BH, MERCHER T, MCDOWELL E, EBERT BL et al. MPLW515L is a novel somatic activating mutation in myelofibrosis with myeloid metaplasia. PLoS Med 2006; e270. doi: 10.1371/journal.pmed.0030270

[13] NANGALIA J, MASSIE CE, BAXTER EJ, NICE FL, GUNDEM G et al. Somatic CALR mutations in myeloproliferative neoplasms with nonmutated JAK2. N Engl J Med 2013; 369: 2391-2405. doi: 10.1056/NEJMoa1312542

[14] KRALOVICS R, PASSAMONTI F, BUSER AS, TEO SS, TIEDT R et al. A gain-of-function mutation of JAK2 in myeloproliferative disorders. N Engl J Med 2005; 352: 17791790. doi: 10.1056/NEJMoa051113

[15] VANNUCCHI AM, ROTUNNO G, BARTALUCCI N, RAUGEI G, CARRAI V et al. Calreticulin mutation-specific immunostaining in myeloproliferative neoplasms: pathogenetic insight and diagnostic value. Leukemia 2014; 28: 18111818. doi: 10.1038/leu.2014.100

[16] DEBRUYN M, WIERSMA VR, HELFRICH W, EGGLETON P, BREMER E. The ever-expanding immunomodulatory role of calreticulin in cancer immunity. Front Oncol 2015; 5: 35. doi: 10.3389/fonc.2015.00035

[17] BAXTER EJ, SCOTT LM, CAMPBELL PJ, EAST C, FOUROUCLAS $\mathrm{N}$ et al. Acquired mutation of the tyrosine kinase JAK2 in human myeloproliferative disorders. Lancet 2005; 365: 1054-1061. doi: 10.1016/S0140-6736(05)71142-9

[18] CHI J, NICOLAUO KA, NICOLAIDOU V, KOUMAS L, MITSIDOU A et al. Calreticulin gene exon 9 frameshift mutations in patients with thrombocytosis. Leukemia 2014; 28:1152-1154. doi: 10.1038/leu.2013.382

[19] CAZZOLA M, KRALOVICS R. From Janus kinase 2 to calreticulin: the clinically relevant genomic landscape of myeloproliferative neoplasms. Blood 2014; 123: 3714-3719. doi: 10.1182/blood-2014-03-530865
[20] RUMI E, PIETRA D, PASCUTTO C, GUGLIELMELLI P, MARTÍNEZ-TRILLOS AM et al. Clinical effect of driver mutations of JAK2, CALR, or MPL in primary myelofibrosis. Blood 2014; 124: 1062-1069. doi: 10.1182/ blood-2014-05-578435

[21] ANDRIKOVICS H, KRAHLING T, BALASSA K, HALM G, BORS A et al. Distinct clinical characteristics of myeloproliferative neoplasms with calreticulin mutations. Haematologica 2014; 99: 1184-1190. doi: 10.3324/haematol.2014.107482

[22] VANNUCCHI AM, LASHO TL, GUGLIELMELLI P, BIAMONTE F, PARDANANI A et al. Mutations and prognosis in primary myelofibrosis. Leukemia 2013; 27: 1861-1869. doi: 10.1038/leu.2013.119

[23] GUGLIELMELLI P, BARTALUCCIN, ROTUNNO G, VANNUCCHI AM. Calreticulin: a new horizon for the testing and treatment of myeloproliferative neoplasms. Expert Rev Hematol 2014; 7: 423-425. doi: 10.1586/17474086.2014.920250

[24] TEFFERI A, WASSIE EA, GUGLIELMELLI P, GANGAT N, BELACHEW AA et al. Type 1 versus Type 2 calreticulin mutations in essential thrombocythemia: a collaborative study of 1027 patients. Am J Hematol 2014; 89: E121-124. doi: 10.1002/ajh.23743

[25] TEFFERI A, LASHO TL, FINKE CM, BELACHEN AA, WASSIE EA et al. Type 1 vs type 2 calreticulin mutations in primary myelofibrosis: differences in phenotype and prognostic impact. Leukemia 2014; 28: 1568-1570. doi: 10.1038/ leu. 2014.83

[26] EDER-AZANZA L, NAVARRO D, ARANAZ P, NOVO FJ, CROSS NC et al. Bioinformatic analyses of CALR mutations in myeloproliferative neoplasms support a role in signaling. Leukemia 2014; 28: 2106-2109. doi: 10.1038/leu.2014.190

[27] TEFFERI A, LASHO TL, FINKE CM, KNUDSON RA, KETTERLING $\mathrm{R}$ et al. CALR vs JAK2 vs MPL-mutated or triple negative myelofibrosis: clinical, cytogenetic and molecular comparisons. Leukemia 2014; 28: 1472-1477. doi: 10.1038/leu.2014.3

[28] PARK SH, KIM SY, LEE SM, YI J, KIM IS et al. Incidence, clinical features, and prognostic impact of CALR exon 9 mutations in essential thrombocythemia and primary myelofibrosis: an experience of a single tertiary hospital in Korea. Ann Lab Med 2015; 35: 233-237. doi: 10.3343/ alm.2015.35.2.233

[29] AHMED RZ, RASHMID M, AHMED N, NEDEEM M, SHAMSI TS. Coexisting JAK2V617F and CALR exon $9 \mathrm{mu}-$ tations in myeloproliferative neoplasms - Do they designate a new subtype? Asian Pac J Cancer Prev 2016; 17: 923-926.

[30] TEFFERI A, LASHO TL, HUANG J, FINKE CM, MESA RA et al. Low JAK2V617F allele burden in primary myelofibrosis, compared to either a higher allele burden or unmutated status, is associated with inferior overall and leukemiafree survival. Leukemia 2008; 22: 756-761. doi: 10.1038/ sj.leu.2405097

[31] BAROSI G, BERGAMASCHI G, MARCHETTI M, VANNUCCHI AM, GUGLIELMELLI P et al. JAK2V617F mutational status predicts progression to large splenomegaly and leukemic transformation in primary myelofibrosis. Blood 2007; 110: 4030-4036. 10.1182/blood-2007-07-099184 
[32] GUGLIELMELLI P, BAROSI G, SPECCHIA G, RAMBALDI A, LOCOCO F et al. Identification of patients with poorer survival in primary myelofibrosis based on the burden of JAK2V617F mutated allele. Blood 2009; 114: 1477-1483. doi: 10.1182/blood-2009-04-216044

[33] LASHO TL, JIMMA T, FINKE CM, PATNAIK M, HANSON CA et al. SRSF2 mutations in primary myelofibrosis: significant clustering with IDH mutations and independent association with inferior overall and leukemia-free survival. Blood 2012; 120: 4168-4171. doi: 10.1182/blood-2012-05-429696

[34] LUNDBERG P, KAROW A, NIENHOLD R, LOOSER R, HAO-SHEN $\mathrm{H}$ et al. Clonal evolution and clinical correlates of somatic mutations in myeloproliferative neoplasms. Blood 2014; 123: 2220-2228. doi: 10.1182/blood-2013-11-537167

[35] TEFFERI A, GUGLIELMELLI P, LASHO TL, ROTUNNO G, FINKE CM et al. CALR and ASXL1 mutations-based molecular prognostication in primary myelofibrosis: an international study of 570 patients. Leukemia 2014; 28: 1494-1500. doi: 10.1038/leu.2014.57
[36] CERVANTES F, DUPRIEZ B, PEREIRA A, PASSAMONTI F, REILLY JT et al. New prognostic scoring system for primary myelofibrosis based on a study of the International Working Group for Myelofibrosis Research and Treatment. Blood 2009; 113: 2895-2901. doi: 10.1182/blood-2008-07-170449

[37] PASSAMONTI F, CERVANTES F, VANNUCCHI AM, MORRA E, RUMI E et al. A dynamic prognostic model to predict survival in primary myelofibrosis: a study by the IWG-MRT (International Working Group for Myelofibrosis Research and Treatment). Blood 2010; 115: 1703-1708. doi: 10.1182/blood-2009-09-245837

[38] GANGAT N, CARAMAZZA D, VAIDYA R, GEORGE G, BEGNA K et al. DIPSS plus: a refined Dynamic International Prognostic Scoring System for primary myelofibrosis that incorporates prognostic information from karyotype, platelet count, and transfusion status. J Clin Oncol 2011; 29: 392397. doi: 10.1200/JCO.2010.32.2446 